www.jmscr.igmpublication.org

Impact Factor 5.84

Index Copernicus Value: 83.27

ISSN (e)-2347-176x ISSN (p) 2455-0450

crossref DOI: https://dx.doi.org/10.18535/jmscr/v5i1.107

Journal Of Medical Science And Clinical Research

IGM Publication

An Official Publication of IGM Publication

\title{
Metronidazole Induced Encephalopathy: Imaging of an Unusual Side Effect
}

\author{
Authors \\ Dr Abhishek Jain ${ }^{1}$, Dr Rajesh Shanklesha ${ }^{2}$, Dr Yadav Shashibala Jayanarayan, \\ Dr Bhakti Yeragi ${ }^{4}$, Dr Gayathri Achuthan ${ }^{5}$ \\ ${ }^{1,5}$ Resident, ${ }^{2}$ Fellow in Body Imaging, ${ }^{3}$ Fellow in Diagnostic Radiology, ${ }^{4}$ Assistant Professor \\ Department of Radio-Diagnosis,T.N.M.C. \& B.Y.L.Nair Charitable Hospital, Mumbai \\ Corresponding Author \\ Dr Abhishek Jain
}

Resident in Radiology, Dept of Radio-Diagnosis, T.N.M.C. \& B.Y.L.Nair CharitableHospital, Mumbai

INDIA

\begin{abstract}
Purpose: Metronidazole is one of the most common drug routinely prescribed for anaerobic and helminthic infections. It is commonly used to treat amebiasis, giardiasis, trichomonas, H.pylori and clostridial infections. Minor side effects like nausea, vomiting, metallic taste in the mouth, headache and abdominal pain may be seen after oral administration. Intravenous administration may cause thrombophlebitis. Rare serious side effects like neutropenia, leukopenia, central nervous system toxicity and peripheral neuropathy can be seen after prolonged and high dose treatment with metronidazole. Many of the times this metronidazole induced complication of central nervoussystem toxicity is under diagnosed due to difficulty in establishing the diagnosis. We here report acase of metronidazole induced encephalopathy and discuss its neuro imaging findings.
\end{abstract}

Case Report: A 50 year old male presented with history of difficulty in walking, mental confusion, slurred speech and double vision. History revealed that he was prescribed metronidazole 50 days back which he has been taking since then (cumulative dose - approximately 60 grams). MRI showed bilaterally symmetrical T2/FLAIR hyper-intensities in dentate nuclei. Other differential diagnosis like wernicke's encephalopathy was ruled by estimation of serum thaimine levels. Metronidazole was discontinued immediately. After discontinuation of metronidazole patient starting improving clinically. A follow up MRI after 20 days of discontinuation of metronidazole showed complete resolution of altered signal intensity areas involving dentate nuclei.

Conclusion: Though rare, metronidazole-induced encephalopathy, is a serious but potentially reversible side effect of prolonged and high dose intake of metronidazole. Acute central nervous system toxicity caused by metronidazole may closely mimic wernicke's encephalopathy. Characterstic neuro imaging findings along with normal thiamine levels confirm the diagnosis of metronidazoleinduced encephalopathy.

Keywords: Metronidazole, Encephalopathy, Neuroimaging, Dentate nuclei, Serum thiamine levels.

\section{Introduction}

Metronidazole is an antimicrobial and antiprotozoalagent that is commonly prescribed for gram negative, helminthic and H.Pylori infections. It is the drug of choice for pseudomembranous colitis caused by clostridium difficle ${ }^{[1]}$. It is routinely used in combination with other antibiotics in intracerebral and abdominal 
abcesses, gynaecological infections and in the preoperative prophylaxis before gynaecological and colorectal surgeries ${ }^{[2]}$. Oral metronidazole intake is associated with minor side effects like nausea, vomiting, metallic taste in the mouth, headache and abdominal pain . Intravenous administration may cause thrombophlebitis ${ }^{[3]}$. Rare serious side effects like neutropenia, leukopenia, central nervous system toxicity and peripheral neuropathy may occur after prolonged intake. metronidazole is believed to cross blood brain barrier and cause central nervous system toxicity in the form of Visual impairment, cerebellar dysfunction in the form of ataxia, nystagmus, dysarthria and irritability ${ }^{[4]}$. In severe cases it may cause encephalopathy manifesting as seizures. Only few cases of metronidazole induced encephalopathy have been reported in literature. The most common symptoms in reported patients have been signs of cerebellar dysfunction, irritability and seizures. The neuroimaging is important in the diagnosis of metronidazole induced encephalopathy because of the characteristic involvement of dentate nucleus (characteristic), midbrain, corpus collasum, medulla, inferior colliculus, basal ganglia and thalamus ${ }^{[5]}$. The lesions present in the form of symmetrical hypo intense lesions on $\mathrm{T} 1$ weighted images and hyperintensities on T2W and FLAIR [6]. since metronidazole induced encephalopathy and its neuroimaging is rare we here report acase of metronidazole induced encephalopathy and discuss its neuroimaging findings.

\section{Case Report}

A 50 year old male patient presented with subacute onset of difficulty in walking, mental confusion, slurring of speech and double vision. The patient has been a chronic alcoholic since 20 years. There was no history of fever, vomitings or trauma in near past. Neither there was history of any major systemic illness like diabetes mellitus or hypertension. General examination, cardiovascular, respiratory and Per abdomen examination was also normal. Examination of central nervous system revealed that the patient was confused and irritable. There was bilaterally symmetrical lower limb weakness. The power of muscles was $5 / 5$ in upper limbs and 3/5 in lower limbs. cerebellar signs like ataxia, dysarthria and dysmetria were present. Renal function tests were normal. Complete blood count showed mild anemia (Hb-11gm \%). Hepatic enzymes were mildly derainged (Mild rise in bilirubin and transaminases). HIV and HbsAg was negative. Given that patient was a known alcoholic a presumptive diagnosis of alcohol withdrawal syndrome, korsakoff psychosis or cerebellar infarct was made by treating physician and the patient was sent to us for neuroimaging. On neuroimaging there were Bilateral symmetrical lesions which were hypo intense on $\mathrm{T} 1$ and hyper intense on T2/FLAIR in dentate nucleus (Fig 1).
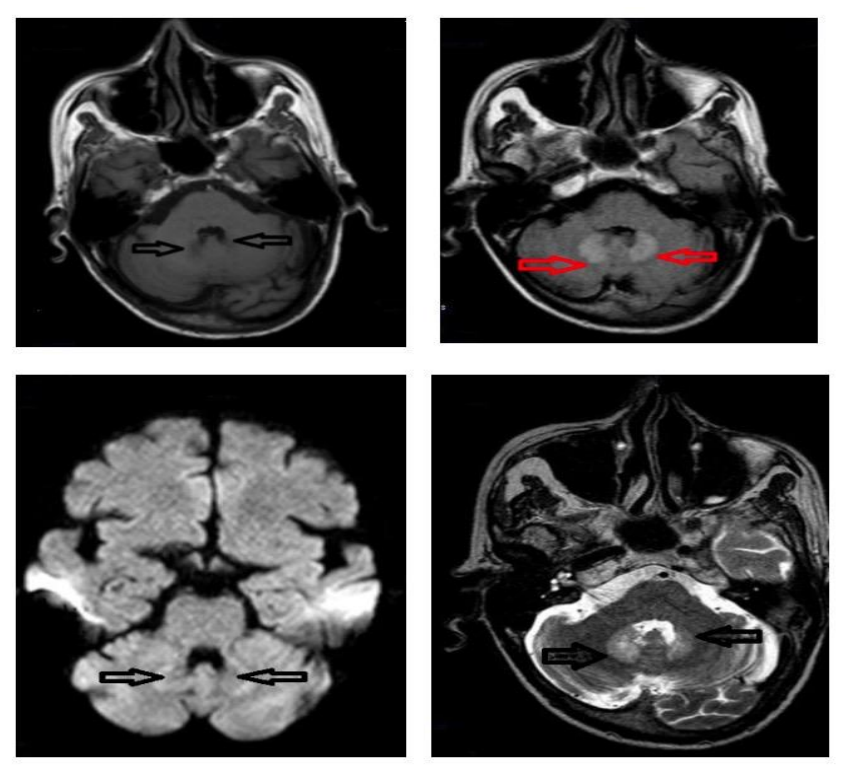

Figure 1 : Characteristic lesions which are hypo intense on $\mathrm{T} 1$ and hyper intense on T2/ FLAIR were seen in dentate nucleus.

Presence of such lesions are rare but unique finding with only few differential diagnoses like Wernicke's encephalopathy, Enteroviral encephalitis, Maple syrup urine disease, drug intoxication like methyl bromide intoxication, metronidazole intoxication. Since patient was a known alcoholic wernike's encephalopathy was considered as primary diagnosis and thiamine levels were done which turned out to be normal. 
Since other differentials were less likely in this patient because maple syrup urine disease would have manifested in childhood and encephalitis would have presented with fever hence drug toxicity as the cause of encephalopathy was contemplated and history was revisited. The drug history was significant as the patient gave history of taking metronidazole in the dose of $400 \mathrm{mg}$ tds since 50 days for recurrent cholangitis (Figure 2).
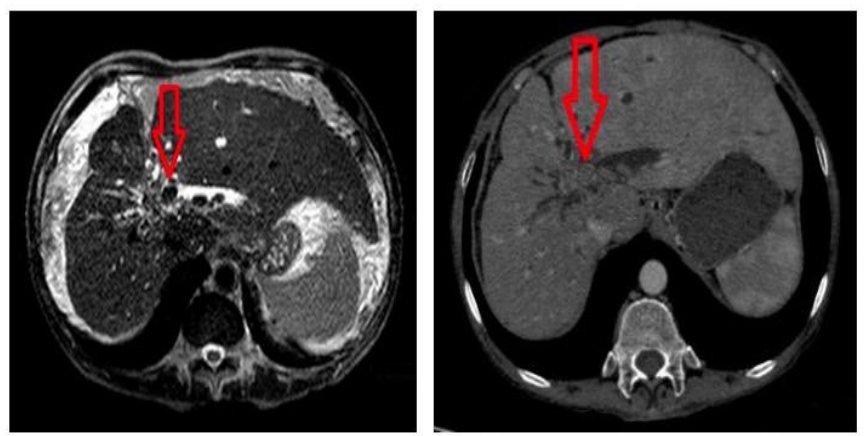

Figure 2: Old CT abdomen suggestive of cholangitis for which patient has been prescribed metronidazole.

The cumulative dose of metronidazole was calculated and was found to be approximately 60 grams. Given the presence of characteristic lesions on neuroimaging with the significant history of metronidazole intake for a prolonged duration a final diagnosis of metronidazole induced encephalopathy was made. Metronidazole was immediately stopped following which the patient started improving clinically. A follow up Scan after 20 days revealed resolution of the lesions in dentate nucleus (Figure 3).
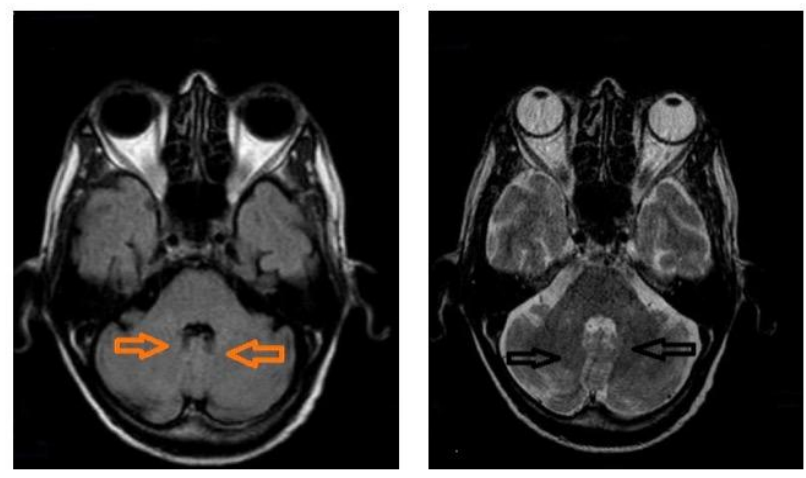

Figure 3 : Follow up MRI after 20 days of stopping metronidazole shows complete resolution of lesions in dentate nucleus.

\section{Discussion}

Metronidazole induced encephalopathy is a rare complication of prolonged intake of metronidazole in excess doses. The exact mechanism of this encephalopathy is not known and it is also not clear why only a small number of patients are affected by characteristic encephalopathy. The fact that many patients were found to be having higher serum levels of metronidazole and no CNS toxicity and some patients developed encephalopathy despite having serum levels of the drug within therapeutic range also goes against the usually held concept that metronidazole causes CNS toxicity if taken in higher doses ${ }^{[7]}$. Various proposed mechanism of encephalopathy include modulation of GABA receptors by the metabolites of metronidazole within the cerebellar and vestibular system and metronidazole's intermediate metabolites modulate inhibitory neurotransmitter GABA receptor especially within the cerebellar and vestibular systems, and generation of semiquinone and neurotoxic radicals due to reaction with catecholamine neurotransmitter ${ }^{[8]}$. Though serum and cerebrospinal fluid level of metronidazole may be obtained to substantiate the diagnosis it is almost certain if characteristic neuroimaging features are present and patient shows clinical improvement after discontinuation of the offending drug ${ }^{[9]}$. Resolution of MRI findings may be an additional proof of the diagnosis being metronidazole induced encephalopathy rather than anything else.

Neuroimaging findings in metronidazole induced encephalopathy are characteristic and was first described by Ahmed et al in $1995^{[10]}$. The characteristic lesions are seen in the dentate nuclei of the cerebellum, corpus callosum, basal ganglia, subcortical white matter. Bilateral lesions which are hypo intense on $\mathrm{T} 1$ and hyper intense on T2/FLAIR are very characteristic of metronidazole induced encephalopathy. These lesions are always bilateral and symmetrical ${ }^{[11]}$.

Treatment usually starts with discontinuation of metronidazole. Though the lesions tend to regress or disappear after discontinuation of metronidazole in some cases these lesion may persist even 
after discontinuation of metronidazole. In such cases persistent encephalopathy and poor outcome is usually expected ${ }^{[12]}$.

\section{Conclusion}

Metronidazole induced encephalopathy is a serious but potentially reversible side effect of metronidazole therapy. An MRI scan of brain should be obtained whenever indicated. The diagnosis can be confirmed if characteristic lesions are present. Resolution of the lesions after discontinuing therapy further confirms the diagnosis in doubtful cases.

\section{Conflict of Interest: None}

\section{References}

1. Shen EP, Surawicz CM. Current Treatment Options for Severe Clostridium difficile-associated Disease. Gastroenterology \& Hepatology. 2008;4(2):134-139.

2. Hansell DT, Thomson GJ, Simpson CJ, Morran C, Smith DC, McNaught W, Gillespie G. Metronidazole prophylaxis in colorectal surgery: the need for additional amino glycoside? J Hosp Infect. 1983 Dec;4(4):383-90.

3. Barnhill AE, Brewer MT, Carlson SA. Adverse Effects of Antimicrobials via Predictable or Idiosyncratic Inhibition of Host Mitochondrial Components. Antimicrobial Agents and Chemotherapy. 2012;56 (8):4046-4051.

4. Sarna JR, Brownell AKW, Furtado S. Reversible cerebellar syndrome caused by metronidazole. CMAJ : Canadian Medical Association Journal. 2009;181(9):611-613.

5. Mulcahy H, Chaddha SKB. MRI of Metronidazole-Induced Encephalopathy. Radiology Case Reports. 2008;3(4):239.

6. Kalia V, Vibhuti, Saggar K. Case report: MRI of the brain in metronidazole toxicity. The Indian Journal of Radiology \& Imaging. 2010;20(3):195-197.

7. Godfrey MS, Finn A, Zainah H, DapaahAfriyie K. Metronidazole-inducedenceph- alopathy after prolonged metronidazole course for treatment of C. difficilecolitis. BMJ Case Rep. 2015 Jan 16;2015.

8. Grill MF, Maganti RK. Neurotoxic effects associated with antibiotic use: management considerations. British Journal of Clinical Pharmacology. 2011;72(3):381393.

9. Roy U, Panwar A, Pandit A, Das SK, Joshi B. Clinical and Neuroradiological Spectrum of Metronidazole Induced Encephalopathy: Our Experience and the Review of Literature. Journal of Clinical and Diagnostic Research: JCDR. 2016;10(6):OE01-OE09.

10. Ahmed A, Loes DJ, Bressler EL. Reversible magnetic resonance imaging findings in metronidazole induced encephalopahty. Neurology. 1995;45:588-9.

11. Kim E, Na DG, Kim EY, Kim JH, Son KR, Chang KH. MR imaging ofmetronidazole-induced encephalopathy: lesion distribution and diffusion-weighted imaging findings. AJNR Am J Neuroradiol. 2007 Oct;28(9):1652-8.

12. Hobbs K, Stern-Nezer S, Buckwalter MS, Fischbein N, Finley Caulfield A Metronidazole-induced encephalopathy: not always a reversible situationNeurocrit Care. 2015 Jun;22(3):429-36. doi: 10.1007/s12028-014-0102-9. 